\title{
Hyperphagia in neonates withdrawing from methadone
}

\author{
Alma Martinez, Beth Kastner, H William Taeusch
}

\begin{abstract}
Aims-To examine whether hyperphagia is a clinically significant problem in infants born to women receiving methadone maintenance.

Methods-The volume of feeds, changes in infant body weight, as well as occurrence of adverse clinical effects in infants withdrawing from methadone were studied during the first month of life. A retrospective chart review was conducted for all infants at San Francisco General between 1992 and 1995, born to women receiving methadone maintenance during their pregnancy. Forty four infants were identified and the data obtained from hospital medical records. The daily oral intake of these infants was recorded during the first month of life. The incidence of hyperphagia (oral intake $>190 \mathrm{cc} / \mathrm{kg} /$ day) was measured. Associations between infant oral intake and maternal methadone dose were studied using correlation analysis as well as Anova for repeated measures. Adverse clinical symptoms were also recorded. A subset of premature infants was studied separately.
\end{abstract}

Results-The incidence of hyperphagia was $26 \%$ by day 8 and $56 \%$ by day 16 of life in the infants. Hyperphagia was not associated with maternal methadone dose or with infant withdrawal scores. Infants who were hyperphagic lost significantly more weight during the first week of life than those who were not. Despite significantly greater intake, the hyperphagic infants did not gain weight more rapidly during the first month of life compared with those infants with lower oral intake. Infants who were hyperphagic (maximum intake of 290 cc/kg/day) did not experience increased vomiting, aspiration, diarrhoea, or abdominal distention.

Conclusions-Hyperphagia is commonly found in infants withdrawing from methadone and can be persistent in a significant number. Hyperphagia was not associated with either increased neonatal weight gain or with adverse gastrointestinal consequences. Hyperphagia may occur in infants withdrawing from methadone who have high metabolic demands due to clinical signs not controlled by opiate treatment.

(Arch Dis Child Fetal Neonatal Ed 1999;80:F178-F182)

Keywords: methadone withdrawal; hyperphagia; metabolism
Excessive oral intake, or hyperphagia, is listed as one of many clinical findings in infants withdrawing from opiates. ${ }^{1-3}$ Conversely, other reports list frantic, disorganised sucking and poor feeding as symptoms of opiate withdrawal. ${ }^{1-8}$ It is unclear from these previous reports how often excessive oral intake is found in infants withdrawing from opiates and whether hyperphagia is associated with adverse clinical consequences for the neonate, such as vomiting, aspiration, diarrhoea, or abdominal distention.

This study aimed to evaluate infants exposed in utero to methadone, to quantify their volume of feeds during inpatient stay, to determine the incidence of hyperphagia in these infants (compared with published norms of feeding patterns in normal neonates), and to quantify weight change as well as the occurrence of adverse clinical effects in infants withdrawing from methadone.

\section{Methods}

Data were collected by retrospective chart review after obtaining approval from the University of California at San Francisco Institutional Review Board for Human Research. The patients were identified from a database of all births at San Francisco General Hospital between 1992 and 1995.

The study population included all infants born at San Francisco General Hospital to women enrolled in a methadone programme during their pregnancy. Infants exposed to other opiates or other illicit drugs in addition to methadone were included in the study. Maternal use of any additional drugs was noted. Infants were treated for signs of withdrawal primarily with tincture of opium, as recommended by the American Academy of Pediatrics. ${ }^{4}$ Diazepam was also used occasionally, but never without the concomitant use of tincture of opium. Infants were excluded from this study if the mother used opiates but was not enrolled in a methadone programme.

All infants in this study remained in hospital after birth for treatment of withdrawal. A significant number of women were prescribed large daily methadone doses, and their infants often required lengthy hospital stays. In the group of infants studied only seven were discharged home before they were 12 days old, and a few remained in hospital for longer than 30 days for management of opiate withdrawal. Data were collected at prospectively chosen time points during the infants' hospital stay (days 8, 12, 16, 20, 24, 28 and 30 of life). Data collected included maternal methadone drug dose, birthweight, gestational age, and daily 


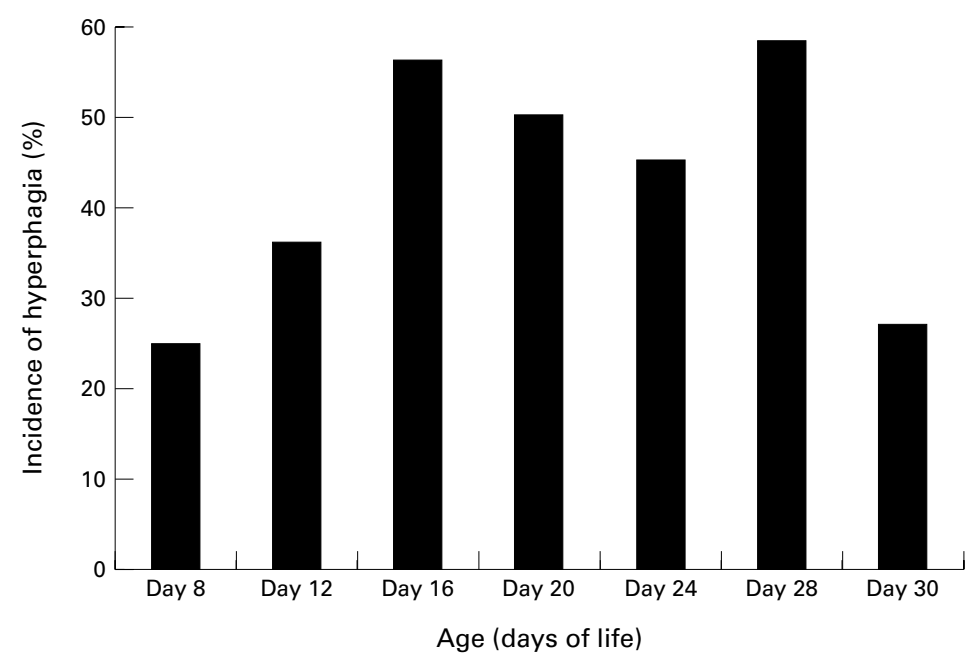

Figure 1 Incidence of hyperphagia (\%) during the first month of life in infants with drawing from methadone. The number of patients remaining in hospital is given in table 1 for each time point. Hyperphagia is defined as a daily oral intake of $>190 \mathrm{cc} / \mathrm{kg} /$ day.

Finnegan withdrawal scores, ${ }^{9}$ assigned by the infant's nurse at each data point. The volume of oral intake, caloric intake, and weight gain were also obtained at the same time points during this period. All infants received infant formula, none was breastfed, and no infant required tube feedings during the study. Drug treatment required for withdrawal was recorded, as were episodes of emesis, drooling, ineffective feeding, diarrhoea, and abdominal distention.

Investigators have carefully documented daily formula intakes for healthy newborn infants living at home. ${ }^{10}$ These investigators have shown that during the first month of life, normal, term infants eat between 140 (SEM 22) $\mathrm{cc} / \mathrm{kg} /$ day and 170 (25) cc/kg/day. Using these previous data, we defined hyperphagia for the purposes of this study, as a daily oral intake greater than $190 \mathrm{cc} / \mathrm{kg} /$ day.

Most values are presented as mean (SEM). Withdrawal scores are shown as median scores and range. Anova for repeated measures was used to compare differences in oral intake between infants exposed to differing methadone doses, and unpaired two-tailed $t$ test was used to compare hyperphagic infants with non-hyperphagic infants for weight gain. The Wilcoxon Rank Sum Test was used to compare withdrawal scores of hyperphagic infants with those of non-hyperphagic infants. Linear regression analysis was performed to deter-

Table 1 Characteristics of infants exposed to methadone

\begin{tabular}{llll}
\hline & All infants $(n=44)$ & $\begin{array}{l}\text { Infants with } \\
\text { hyperphagia }\end{array}$ & $\begin{array}{l}\text { Infants without } \\
\text { hyperphagia }\end{array}$ \\
\hline $\begin{array}{l}\text { Methadone dose } \\
\text { Median withdrawal scores }\end{array}$ & $50+3$ & $51(5)$ & $57(6)$ \\
Oral intake $(c c / k / d):$ & 11 & 11 & \\
Day 8 & $165(7)(\mathrm{n}=44)$ & $222(6)^{\star}(\mathrm{n}=11)$ & $145(6)(\mathrm{n}=31)$ \\
Day 12 & $175(7)(\mathrm{n}=36)$ & $223(6)^{\star}(\mathrm{n}=13)$ & $147(5)(\mathrm{n}=23)$ \\
Day 16 & $192(8)(\mathrm{n}=30)$ & $221(8)^{\star}(\mathrm{n}=17)$ & $154(5)(\mathrm{n}=13)$ \\
Day 20 & $202(7)(\mathrm{n}=26)$ & $233(6)^{\star}(\mathrm{n}=13)$ & $171(5)(\mathrm{n}=13)$ \\
Day 24 & $197(8)(\mathrm{n}=22)$ & $226(8)^{\star}(\mathrm{n}=10)$ & $169(5)(\mathrm{n}=12)$ \\
Day 28 & $201(10)(\mathrm{n}=17)$ & $229(9)^{\star}(\mathrm{n}=10)$ & $162(5)(\mathrm{n}=7)$ \\
Day 30 & $183(10)(\mathrm{n}=15)$ & $218(16)^{\star}(\mathrm{n}=4)$ & $170(3)(\mathrm{n}=11)$ \\
\hline
\end{tabular}

Data are shown as mean (SEM). The number of infants at each time point who remained in hospital is noted $(n=) .^{\star}$ significant difference in the oral intake between the hyperphagic infants and those with normal intake $(\mathrm{p}<0.05$; Anova). mine the predictive value of maternal methadone dose, birthweight, and gestational age to oral intake or to withdrawal scores. The data were analysed using the True Epistat Data Analysis Program (Richardson, Texas). The level of statistical significance was chosen as $\mathrm{p}<0.05$.

\section{Results}

Forty four infants were identified during the study period who were born to women enrolled in a methadone maintenance programme. Thirty nine per cent of these women were polydrug users, with $60 \%$ also using heroin. Fifty five per cent of the women reported using cocaine or tested positive during the current pregnancy, and $10 \%$ had a history of alcohol use during the current pregnancy.

The mean birthweight for all infants studied was 2804 (112) $\mathrm{g}$ (range 1340 to 4100 ). The mean gestational age was $37(0.5)$ weeks (range 30 to 42 ). The mean daily maternal methadone dose was 50 (3.2) $\mathrm{mg}$ (range 15 to 95). The formula oral intake for all infants as well as the number of infants studied at each time point studied is shown in table 1 . By day 20 of life, the mean oral intake of infants withdrawing from methadone, had reached $202 \mathrm{cc} / \mathrm{kg} /$ day. Using previous data for normal formula intake of healthy, term infants, we defined hyperphagia as a daily oral intake greater than $190 \mathrm{cc} / \mathrm{kg} /$ day. Using these criteria, $26 \%$ of the infants were hyperphagic by day 8 of life, and $56 \%$ by day 16 of life. (fig 1 ).

Using a multiple linear regression model, we found no significant correlation between infant oral intake at any time period studied and maternal methadone dose at delivery, birthweight, or gestational age (on day 8 of life, $r=$ $0.27, \mathrm{p}=0.4$ ). We stratified the infants into three groups of methadone exposure, based on daily maternal dose. The "low" dose of exposure was defined as $0-30 \mathrm{mg}$ daily, "medium" as $35-50 \mathrm{mg}$ daily, and "high" as 55-95 mg daily. Using these stratified data, we found no difference in the daily intake between the infants born to mothers with low, medium, or high methadone doses at each time point measured ( $p>0.05$, Anova) (fig 2).

As shown in fig 3, despite significantly greater oral intake in hyperphagic infants than in infants with lower oral intake, the hyperphagic infants lost significantly more weight during the first week of life. During the rest of their hospital stay, the hyperphagic infants did not show differences in weight gain when compared to the lower oral intake group (fig 3).

Withdrawal scores of infants who were hyperphagic did not differ from infants who had lower levels of oral intake except for day 28 of life (Wilcoxon Rank Sum Test). At this late date, the median withdrawal score for hyperphagic infants was 12 compared with five for non-hyperphagic infants; $\mathrm{p}=0.03$.

There were no increased gastrointestinal symptoms, such as vomiting, diarrhoea, drooling, abdominal distention or aspiration found in infants who were hyperphagic compared with infants with lower oral intake $(p>0.05$; 


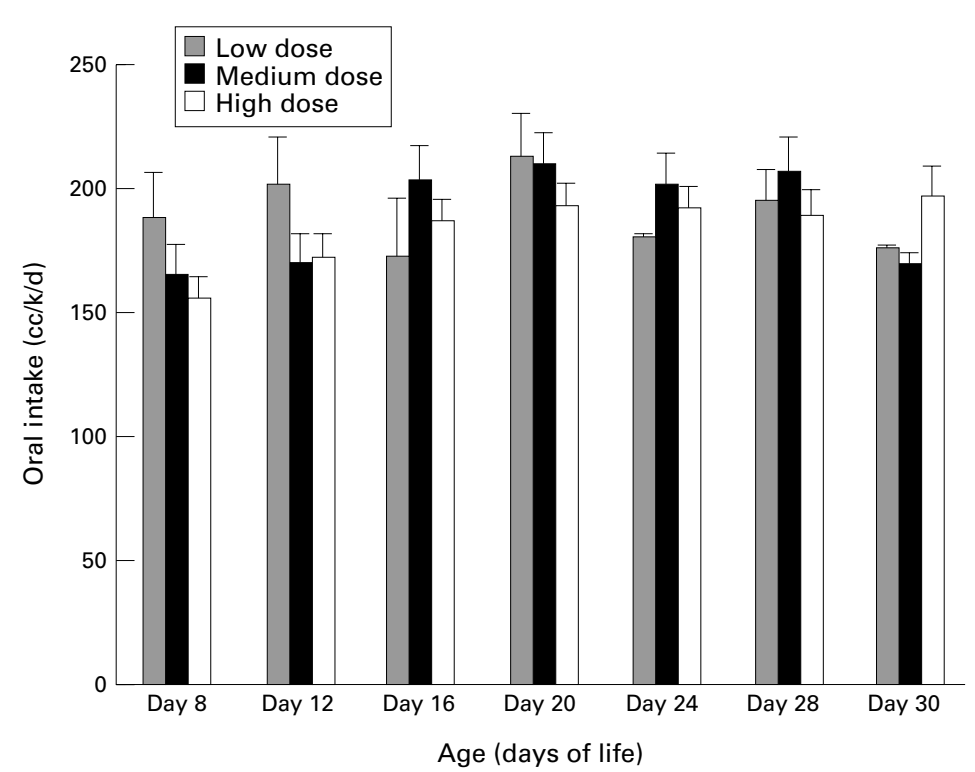

Figure 2 Formula intake in infants withdrawing from methadone during the first month of life. Oral intake was stratified by maternal methadone dose. Comparisons between low dose (<30 mg/day), medium dose (35-50 mg/day), and high dose (55-95 mg/day) are shown. Values are mean (SEM); $p>0.05$, Anova.

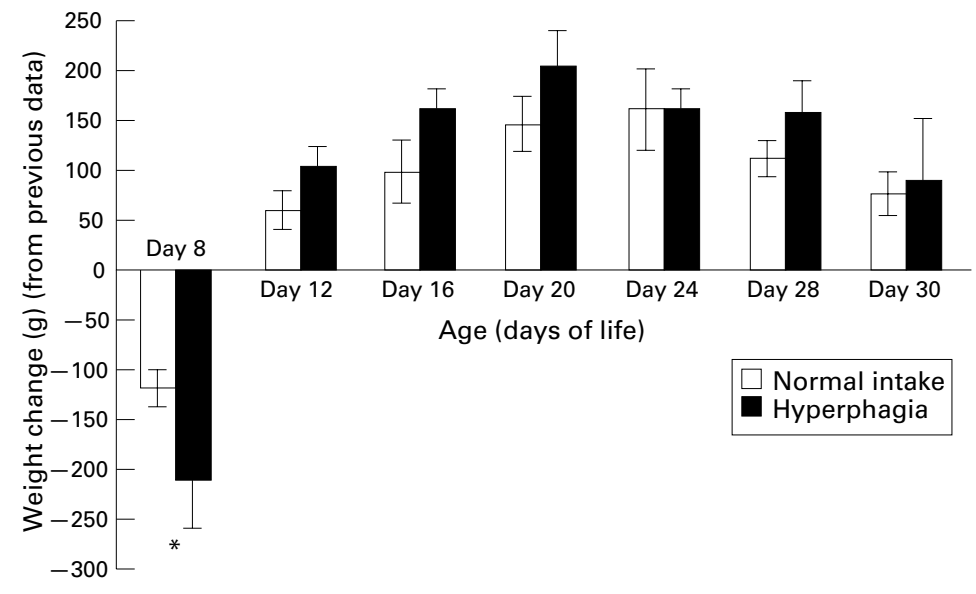

Figure 3 Weight change (g/day), as measured from the previous time point, for infants withdrawing from methadone during the first month of life. Comparisons made between infants with normal intake vs high (hyperphagia) intake; ${ }^{\star} p<0.05$, Anova.

Anova). Contrary to our expectations, no hyperphagic infant had a clinical complication attributable to increased oral intake.

Fifteen preterm infants were included in this study. The patient characteristics of the preterm infants are shown in table 2 . The mean gestational age for the preterm infants was 33 weeks (range 30-36), the mean birthweight was $2136 \mathrm{~g}$ (range 1340-3365). There was no statistical difference between the maternal methadone dose in preterm or term infants. The oral intake for these preterm infants was also calculated and analysed separately to investigate whether there were significant differences in clinical outcomes or feeding intake for these smaller infants. There was only one infant who had low intake $(56 \mathrm{cc} / \mathrm{kg} /$ day) during the first week of life. This was the smallest infant in the study, and in this instance, the infant's oral intake was deliberately limited by the physicians caring for the child. Despite prematurity in this subset of infants, there were no statistical differences in
Table 2 Characteristics of preterm infants exposed to methadone

\begin{tabular}{lll}
\hline & \multicolumn{2}{l}{ Preterm infants } \\
\cline { 2 - 3 } & $(n=15)$ & Range \\
\hline Birthweight (g) & $2136(138)$ & $1340-3365$ \\
Gestational age (weeks) & $33.4(0.4)$ & $30-36$ \\
Methadone dose (mg) & $47.4(3)$ & $30-95$ \\
Oral intake (cc/k/d): & $178(21)$ & \\
Day 8 & $171(17)$ & \\
Day 12 & $182(21)$ & \\
Day 16 & $181(17)$ & \\
Day 20 & $177(14)$ & \\
Day 24 & $183(21)$ & \\
Day 28 & $170(5)$ & \\
Day 30 & \\
\hline
\end{tabular}

All newborn data shown are mean (SEM). For each time point, there was no significant difference between oral intake for premature infants $v$ s term infants ( $\mathrm{p}>0.05$; Anova).

oral intake or clinical feeding problems in the preterm infants when compared with term infants at any time point ( $p>0.05$; Anova).

\section{Discussion}

While hyperphagia or excessive oral intake has been cited before as a clinical finding in neonates undergoing opiate withdrawal, the actual incidence of this finding has not been reported. ${ }^{1-3}$ Hyperphagia is common in infants withdrawing from methadone, occurring in $56 \%$ of infants by the second week of life. Infants with hyperphagia lost significantly more weight during the first week of life, and had similar weight gains thereafter, when compared with non-hyperphagic infants withdrawing from methadone. No significant correlation between maternal methadone dose and infant oral intake or withdrawal scores was found during the first month of life. The infants who were hyperphagic did not have a greater incidence of ineffective feeding or sucking, vomiting, or diarrhoea. A smaller subset of preterm infants was studied separately and they were no different from full term infants with regards to all outcomes measured for this study.

Excessive sucking or disorganised and ineffectual sucking associated with poor oral intake has been reported before in infants withdrawing from opiates. ${ }^{4-8}$ We found the oppositethat is, by the second week of life, a large percentage of infants are hyperphagic. In our study there was only one infant who had low oral intake $(56 \mathrm{cc} / \mathrm{kg} /$ day $)$ during the first week of life. This was the smallest infant studied and in this instance, the infant's oral intake was deliberately limited by the physicians caring for the child. Beginning in the second week of life, this infant had increased oral intake that continued for the rest of his inpatient stay. The lack of ineffectual feeding in this cohort of infants withdrawing from methadone is an interesting finding. Perhaps the use of neonatal scoring systems to quantify the severity of withdrawal, as well as the prompt use of medications to control withdrawal, enable these infants to eat effectively.

The use of a historical control group for this study is a serious consideration in evaluating these findings. The elegant data published by previous workers detailing the normal infant 
oral intake would be costly and difficult to replicate. ${ }^{10}$ These investigators followed a cohort of healthy, term infants for 120 days, and measured formula intake, caloric intake, and growth parameters. It would also not be possible to use a "healthy" hospital control group of infants, as healthy infants are not admitted for lengthy periods as were these infants withdrawing from methadone. This remains an acknowledged limitation of the current study. A natural extension for future studies would be to look at the feeding patterns of infants similarly treated for withdrawal who are exposed to other opiates.

The question of whether large volumes of oral intake are harmful for these infants was a primary reason for the initiation of this study. We found no evidence of adverse gastrointestinal clinical occurrences in the term or preterm hyperphagic infants. The finding of greater weight loss in the first four days of life followed by similar weight gains in the hyperphagic infants compared with the infants on lower oral intake, suggests higher metabolic demands (or greater caloric losses) in the hyperphagic infants. Perhaps administration of higher doses of medications in hyperphagic infants might lead to lower oral intake as well as more effective treatment of other signs of withdrawal.

Our finding of a lack of correlation between maternal methadone dose and infant's oral intake, as well as a lack of correlation between withdrawal scores and hyperphagia during the first month of life, is consistent with results from some previous studies but disagrees the work of others. Mack et al found no correlation between neonatal serum concentrations of methadone and the severity of withdrawal in the neonates. ${ }^{11}$ Previous workers similarly found no correlation with neonatal withdrawal and maternal methadone dose. ${ }^{12}$ Conversely, others have found a significant correlation between the severity of neonatal withdrawal and maternal methadone dose. ${ }^{13}$ These same authors, however, found no correlation found between neonatal serum concentrations and the severity of withdrawal in the infant. ${ }^{13}$ Doberczak and colleagues reported that clinical signs of withdrawal correlated with the rate of decline of the neonatal plasma drug concentrations during the first few days of life. ${ }^{14}$ Lastly, others have reported that the need for medical treatment to infants after birth is highest in those born to mothers receiving higher doses of methadone at delivery. ${ }^{15-17}$ These divergent findings have been used to argue for either weaning pregnant women to a low methadone maintenance dose ${ }^{141517}$ to attempt complete maternal detoxification during pregnancy, ${ }^{16}$ or for maintaining the maternal methadone dose throughout the pregnancy to avoid fetal distress and death associated with maternal detoxification and withdrawal. ${ }^{4} 111819$ The medical management of pregnant women addicted to opiates remains controversial.

The incidence of low birthweight and prematurity found in this study population seems similar to results reported before. The mean birthweight of the infants in this current study was $2804 \mathrm{~g}$, with $33 \%$ of infants weighing less than $2500 \mathrm{~g}$ at birth. This supports previous observations of a 25 to $50 \%$ incidence of low birthweight associated with opiate exposure during gestation. ${ }^{482023}$

Most of the infants in this study were exposed to multiple illicit drugs during gestation, and we acknowledge that the results in this paper can not be solely attributed to the effects of maternal methadone use. The problem of multiple drug exposure in utero continues to be a significant limitation in interpreting the results of clinical research in this area. As many investigators have shown, women who use illicit drugs during pregnancy frequently expose themselves to multiple drugs. In a recent study from the Netherlands, over $56 \%$ of women in a methadone programme had used other opiates, and $57 \%$ had used cocaine. ${ }^{24}$ Findings such as these preclude clinical investigations of infants exposed to only one illicit drug during pregnancy. Exposure to additional drugs may have affected the infants in this study by adding to their clinical signs. Infants exposed to methadone alone, or in combination with other drugs during gestation, might show qualitative and/or quantitative differences in the clinical signs of withdrawal as well as in other neonatal characteristics, as has been described before by others. ${ }^{21}{ }^{25}$

In summary, hyperphagia was common among a group of infants withdrawing from methadone. By the second week of life, over half of the infants studied were eating more than $190 \mathrm{cc} / \mathrm{kg} /$ day. The hyperphagia seemed to be well tolerated by infants, and there were no increased adverse clinical findings associated with high intake in this relatively small number of infants. The infants who exhibited hyperphagia lost more weight in the first few days of life and also did not show greater weight gain than other infants when examined in the first month of life. We did not find that any infants had poor or ineffectual feeding. Further studies should be undertaken to determine whether it is beneficial for infants withdrawing from opiates to have medical management of hyperphagia, and whether this can be achieved without adversely affecting hospital stay.

1 Ostrea E, Lucena J, Silvestre M. The infant of the drug-dependent mother. In: Avery $G$, Fletcher $M$, MacDonald M, editors. Neonatology. Fourth Edn. Philadephia: JB Lippincott Company, 1994:1300-5

2 Hutchings D, Dow-Edwards D. Animal models of opiate, cocaine, and cannabis use. Clin Perinatol 1991;18:1-22.

3 Rothstein P, Gould J. Born with a habit. Pediatr Clin North Am 1974;21:307-21.

4 American Academy of Pediatrics. Committe on Drugs. Neonatal drug withdrawal. Pediatr 1983;72:895-7.

5 Anand $\mathrm{K}$, Arnold J. Opioid tolerance and dependence in infants and children. Crit Care Med 1994;22:334-42.

6 Levy M, Spino M. Neonatal withdrawal syndrome: Associated drugs and pharmacologic management. Pharmacotherapy 1993;13:202-11.

7 Franck L, Vilardi J. Assessment and management of opioid withdrawal in ill neonates. Neonatal Network 1995;14:39-47. 8 Lam S, To W, Duthie S, Ma H. Narcotic addiction in pregnancy with adverse maternal and perinatal outcome. Aust NZ F Obstet Gynaecol 1992;32:216-21.

9 Finnegan L, Kaltenbach K. Neonatal abstinence syndrome. In: Primary Pediatric Care. 3rd Edn. Hoekelman RA, et al, eds. St Louis: CV Mosby, 1992:1367-8.

10 Fomon S, Thomas L, Filer L, Ziegler E, Leonard M. Food consumption and growth of normal infants fed milk-based formulas. Acta Paediatrica Scand (Suppl) 1971;223:1-30.

11 Mack G, Thomas D, Giles W, Buchanan N. Methadone levels and neonatal withdrawal. $\mathcal{f}$ Paediatr Child Health 1991;27:96-100. 
12 Rosen T, Pippenger C. Pharmacologic observations on the neonatal withdrawal syndrome 7 Pediatr the neonatal

13 Harper R, Solish G, Feingold E, Gersten-Woolf N, Sokal M Maternal ingested methadone, body fluid methadone, and the neonatal withdrawal syndrome. Am $\mathcal{f}$ Obstet Gynecol 1977;129:417-24.

14 Doberczak T, Kandall S, Friedmann P. Relationships between maternal methadone dosage, maternal-neonatal methadone levels and neonatal withdrawal. Obstet Gynecol 1993;81:936-40.

15 Kandall S, Doberczak T, Mauer K, Strashun R, Korts D. Opiate vs CNS depressant therapy in neonatal drug abstinence syndrome. Am $\mathcal{F}$ Dis Child 1983;137:378-82.

16 Mass U, Kattner E, Weingart-Jesse B, Schafer A, Obladen $M$. Infrequent neonatal opiate withdrawal following maternal methadone detoxification during pregnaning Perinatal Med 1990;18:111-18

17 Madden J, Chappel J, Zuspan F, Gumpel J, Mejia A, Davis R R. Observation and treatment of neonatal narcotic
withdrawal. Am f Obstet Gynecol 1977;127:199-201.
18 Zuspan F, Gumpel J, Mejia-Zelaya A, Madden J, Davis R. Fetal stress from methadone withdrawal. Am $\mathcal{F}$ Obstet Fetal stress from methad

19 Barr G, Jones K. Opiate withdrawal in the infant. Neurotoxicol Teratol 1994;16:219-25.

20 Chiriboga C. Fetal effects. Neurologic Clinics 1993;11:70727.

21 Chasnoff I, Hatcher R. Burns W. Polydrug- and methadoneaddicted newborns: A continuum of impairment? Pediatrics 1982;70:210-13.

22 Ostrea E, Chavez C. Perinatal problems (excluding neonatal withdrawal) in maternal drug addiction: A study of 830 cases. F Pediatr 1979;94:292-5.

23 Hoegerman G, Schnoll S. Narcotic use in pregnancy. Clin Perinatol 1991;18:51-76.

24 Boer K, Smit B, van Huis A, Hogerzeil H. Substance use in pregnancy: Do we care? Acta Paediatr Suppl 1994;404:65-

5 Fulroth R, Phillips B, Durand D. Perinatal outcome of infants exposed to cocaine and/or heroin in utero. $A m \mathcal{F}$ Dis Child 1989;143:905-10. 